\title{
The importance of the White Book of Radiation Oncology
}

\author{
Ferran Guedea $\cdot$ Alfredo Ramos
}

$\mathrm{T}$ he current issue of Clinical and Translational Oncology includes an article that summarises and contextualises the recently published Libro Blanco de la Oncología Radioterápica, a book written by members of the Spanish Society of Radiation Oncology (SEOR). The White Book of Radiation Oncology is the first of its kind in Spain and its importance cannot be overstated.

The White Book consists of 13 chapters on a wide range of topics that cover all aspects of radiation oncology and its role in cancer care in Spain. The aim of the book is to give a comprehensive overview of the current state of radiation oncology in Spain and to serve as a reference for physicians, health care administrators and hospital managers.

Although it may be hard to remember a time when radiation oncology did not exist as a distinct speciality, it was only just over three decades ago (in 1978, to be precise) that the Association of Radiotherapy and Oncology (AERO) was created. In 2006, the name of the association was changed to SEOR to reflect the fact that specialists in our field are oncologists first and radiotherapy experts second. The SEOR has grown significantly since its founding and now has more than 750 members. In addition, the SEOR is also active internationally and has signed numerous agreements for cooperation with other medical societies, including the European Society for Therapeutic Radiology and Oncology (ESTRO).

The rapid technological progress that has been made in the last decade allows us to deliver radiation with increasing precision and effectiveness, thus increasing the number of tumour localisations amenable to treatment with radiation therapy. As a result, approximately half of all cancer patients are now treated with radiotherapy. While

F. Guedea (西)

Department of Radiation Oncology

Catalan Institute of Oncology

Gran vía, s/n - km 2,7

ES-08907 L'Hospitalet de Llobregat, Barcelona, Spain

e-mail: guedea@iconcologia.net

\section{A. Ramos}

Department of Radiation Oncology

Hospital Ramón y Cajal

Madrid, Spain

these technological advances are clearly welcome, they also present new challenges, as specialists must constantly strive to keep up to date with the latest techniques and equipment. Technologies that seemed cutting edge only a few years ago, for example intensity-modulated radiotherapy (IMRT), are now considered standard treatment for certain tumours. Still, many of the newer technologies-4D radiotherapy, image-guided RT (IGRT), breathing-synchronised RT, SBRT, radiosurgery and intraoperative RT (IORT)-have yet to be installed in smaller clinics, even as they become increasingly common in larger RT centres. In Spain, radiotherapy centres tend to purchase and incorporate "new" machinery slowly (for a myriad of reasons, not least of which is cost) and it seems clear that we need to find ways to accelerate the purchase of newer, more effective technologies for the benefit of our patients.

Even as we recognise the importance of state of the art technologies, it is important to remember that we are physicians first and our main priority is to provide the patient with the best care possible. Quality care in our field depends not only on first-rate technology, but also on the skill and training of the specialists responsible for treating patients. Radiation oncologists must be experts in both the technical and clinical aspects of radiotherapy.

Cancer treatment in general has become more interdisciplinary, thus requiring the cooperation of a larger number of specialists. The growth of tumour boards, where complex cases are discussed, is a prime example. It is during these meetings that each specialist can share his/her specialised knowledge so that a well founded treatment decision can be made.

Important changes have also occurred in training new specialists (residency) and continuing education for current staff. Spain, along with all other member countries of the European Union, has had to reorganise its university curriculum to bring it in line with the new European standards as established by the Bologna Agreement. As a result, students must now complete 360 European credits (9000 hours) to obtain a degree in medicine. Although the current transition period has been challenging, it also offers new opportunities for our field, and several universities have proposed new courses that combine aspects of both medical and radiation oncology. These courses are intended for the fifth or sixth year of the degree programme. 
Perhaps the biggest issue confronting us now in training new specialists is the current 4-year residency requirement, which falls short of the recommendations proposed by the European Core Curriculum on Radiotherapy (which calls for a 5-year training programme). Since 2 of the current 4 years will be focused on core medical training, there is little time for clinical radiation oncology. A proposal to increase the residency to 5 years has already been submitted to the Ministry of Health and we are currently awaiting a response. Unfortunately, it is not at all clear that this proposal will be accepted, and it may be necessary to press our case more vigorously.

In 2008, the Spanish School of Radiation Oncology (EEOR) was created by the SEOR to provide its members with continuing education courses in oncology, radiobiology, molecular biology and radiotherapy. The EEOR has grown rapidly due to the strong demand for courses to help both residents and attending physicians keep up to date with the ever-increasing progress in technology and medical knowledge. We believe that much of the success of the EEOR can also be attributed to the high quality and professionalism of the EEOR staff and professors.

Much progress has been made in both basic and clinical research in recent years, yet more needs to be done. We must find ways to strengthen participation in preclinical research projects and to improve training in radiobiology for future specialists. Clinical research has a strong tradition in Spain and numerous working groups have been formed through the SEOR to perform and coordinate this type of research. Most of these groups focus on a single pathological location (prostate, breast) or have a very well defined area of interest (such as the Young Radiation Oncologists group). Likewise, members of the SEOR are increasingly involved in national and international interdisciplinary groups, such as GEICAM (breast cancer), GENO (neurooncological cancers) and, of course, the largest and most important European research group in our field, the EORTC (European Organisation for Research and Treatment of Cancer). Despite clear progress in both basic and clinical research, there is still room for improvement in both areas.

In conclusion, we believe that the Spanish White Book of Radiation Oncology represents an important milestone in the history of our speciality in Spain. The report is remarkably comprehensive and we believe that it should be required reading for all professionals involved in cancer care in this country. The article published in this issue provides a good overview of the book and we strongly recommend it to all Clinical and Translational Oncology readers. 